\title{
Symmetries of Dynamically Equivalent Theories
}

\author{
D. M. Gitman and I. V. Tyutin \\ Institute of Physics, University of São Paulo, Brazil and \\ Lebedev Physics Institute, Moscow, Russia
}

Received on 31 January, 2005

\begin{abstract}
A natural and very important development of constrained system theory is a detail study of the relation between the constraint structure in the Hamiltonian formulation with specific features of the theory in the Lagrangian formulation, especially the relation between the constraint structure with the symmetries of the Lagrangian action. An important preliminary step in this direction is a strict demonstration, and this is the aim of the present article, that the symmetry structures of the Hamiltonian action and of the Lagrangian action are the same. This proved, it is sufficient to consider the symmetry structure of the Hamiltonian action. The latter problem is, in some sense, simpler because the Hamiltonian action is a first-order action. At the same time, the study of the symmetry of the Hamiltonian action naturally involves Hamiltonian constraints as basic objects. One can see that the Lagrangian and Hamiltonian actions are dynamically equivalent. This is why, in the present article, we consider from the very beginning a more general problem: how the symmetry structures of dynamically equivalent actions are related. First, we present some necessary notions and relations concerning infinitesimal symmetries in general, as well as a strict definition of dynamically equivalent actions. Finally, we demonstrate that there exists an isomorphism between classes of equivalent symmetries of dynamically equivalent actions.
\end{abstract}

Keywords: Constrained systems; Symmetries; Lagrangian actions

\section{INTRODUCTION}

The most of contemporary particle-physics theories are formulated as gauge theories. It is well known that within the Hamiltonian formulation gauge theories are theories with constraints. This is the main reason for a long and intensive study of the formal theory of constrained systems, see [1]. It still attracts considerable attention of researchers. From the very beginning, it became clear that the presence of firstclass constraints among the complete set of constraints in the Hamiltonian formulation is a direct indication that the theory is a gauge one, i.e., its Lagrangian action is invariant under gauge transformations. A next natural, and very important, step would be a detail study of the relation between the constraint structure and constraint dynamics in the Hamiltonian formulation with specific features of the theory in the Lagrangian formulation, especially the relation between the constraint structure with the gauge transformation structure of the Lagrangian action. An important problem to be solved in this direction would be a strict demonstration, and this is the aim of the present article, that the symmetry structures of the Hamiltonian action and of the Lagrangian action are the same. This proved, it is sufficient to consider the symmetry structure of the Hamiltonian action. The latter problem is, in some sense, simpler because the Hamiltonian action is a firstorder action. At the same time, the study of the symmetry of the Hamiltonian action naturally involves Hamiltonian constraints as basic objects, see $[2,3]$. It follows from the results of the article [4] that the Lagrangian and Hamiltonian actions are dynamically equivalent. This is why in the present article we consider from the very beginning a more general problem: how the symmetry structures of dynamically equivalent actions are related. The article is organized as follows: In sec. 2 , we present some necessary notions and relations concerning infinitesimal symmetries in general. A strict definition of dynamically equivalent actions is given in sec. 3. Finally, in sec. 4, we demonstrate that there exists an isomorphism between classes of equivalent symmetries of dynamically equivalent actions.

\section{SYMMETRIES}

\section{A. Basic notation and relations}

We consider finite-dimensional systems which are described by the generalized coordinates $q \equiv\left\{q^{a} ; a=\right.$ $1,2, \ldots, n\}$. The space of the variables $q^{a[l]}$,

$$
q^{a[l]}=\left(d_{t}\right)^{l} q^{a}, l=0,1, \ldots, N_{a}, \quad\left(q^{a[0]}=q^{a}\right), d_{t}=\frac{d}{d t},
$$

considered as independent variables, with finite $N_{a}$, or with some infinite $N_{a}$, is called the jet space. The majority of physical quantities are described by so-called local functions (LF) which are defined on the jet space. The LF depend on $q^{a[l]}$ up to some finite orders $l \leq N_{a} \geq 0$. The following notation is often used[6]:

$$
F\left(q^{a[0]}, q^{a[1]}, q^{a[2]}, \ldots\right)=F\left(q^{[]}\right)
$$

for the LF. In what follows, we also deal with so-called local operators (LO). LO $\hat{U}_{A a}$ are matrix operators which act on columns of LF $f^{a}$ producing columns $F^{A}$ of LF, $F^{A}=\hat{U}_{A a} f^{a}$. LO have the form

$$
\hat{U}_{A a}=\sum_{k=0}^{K<\infty} u_{A a}^{k}\left(d_{t}\right)^{k}
$$

where $u_{A a}^{k}$ are LF. We call the operator 


$$
\left(\hat{U}^{T}\right)_{a A}=\sum_{k=0}^{K<\infty}\left(-d_{t}\right)^{k} u_{A a}^{k}
$$

the transposed operator with respect to $\hat{U}_{A a}$. The following relation holds true for any $\operatorname{LF} F^{A}$ and $f_{a}$ :

$$
F^{A} \hat{U}_{A a} f^{a}=\left[\left(\hat{U}^{T}\right)_{a A} F^{A}\right] f^{a}+d_{t} Q,
$$

where $Q$ is an LF. The LO $\hat{U}_{a b}$ is symmetric (+) or antisymmetric (-) respectively if $\left(\hat{U}^{T}\right)_{a b}= \pm \hat{U}_{a b}$. Thus, for any antisymmetric LO $\hat{U}_{a b}$ relation (5) is reduced to the following: $f^{a} \hat{U}_{a b} f^{b}=d Q / d t$, where $Q$ is a LF.

Suppose the total time derivative of an LF vanishes. Then this LF is a constant. Namely,

$$
\frac{d F\left(q^{[l]}(t)\right)}{d t} \equiv 0 \Longrightarrow F\left(q^{[l]}\right) \equiv \text { const } .
$$

Indeed, let us suppose that $N_{a}$ are the orders of the coordinates $q^{a}$ in the LF, i.e. $F\left(q^{[l]}\right)=F\left(\cdots q^{a\left[N_{a}\right]}\right)$. Then according to (6) the following relation holds true

$$
\frac{\partial F}{\partial q^{a\left[N_{a}\right]}} q^{a\left[N_{a}+1\right]} \equiv-\left[\partial_{t} F+\sum_{a} \sum_{k=0}^{N_{a}-1} \frac{\partial F}{\partial q^{a[k]}} q^{a[k+1]}\right] .
$$

The right hand side of the above relation does not depend on $q^{a\left[N_{a}+1\right]}$. Thus, $\partial F / \partial q^{a\left[N_{a}\right]} \equiv 0$, and therefore $F\left(q^{[l]}\right)$ must not depend on $q^{a\left[N_{a}\right]}$. In the same manner we can see that $F\left(q^{[l]}\right)$ must not depend on $q^{[N-1]}$ and so on. If $F\left(q^{[l]}\right)$ does not depend on any $q^{[l]}$, then $\partial_{t} F\left(q^{[l]}\right) \equiv 0$ as well, and we get $F\left(q^{[l]}\right)=$ const.

We recall that $F_{A}\left(q^{\square}\right)=0$ and $\chi_{\alpha}\left(q^{[}\right)=0$ are equivalent sets of equations whenever they have the same sets of solutions. In what follows, we denote this fact as $F=0 \Longleftrightarrow \chi=$
0 . Via $O(F)$ we denote any LF that vanishes on the equations $F_{a}\left(q^{\square}\right)=0$. More exactly, we define $O(F)=\hat{V}^{b} F_{b}$, where $\hat{V}^{b}$ is an LO. Besides, we denote via $\hat{U}=\hat{O}(F)$ any LO that vanish on the equations $F_{a}\left(q^{\square}\right)=0$. That means that the LF $u$ that enter into (3) vanish on these equations, $u=O(F)$, or equivalently $\hat{U} f=O(F)$ for any $\operatorname{LF} f$.

We consider Lagrangian theories given by an action $S[q]$,

$$
S[q]={ }_{t_{1}}^{\mathrm{Z}_{2}} L d t, L=L\left(q^{[]}\right),
$$

where a Lagrange function $L$ is defined as an LF on the jet space[7]. The Euler-Lagrange equations are

$$
\frac{\delta S}{\delta q^{a}}=\sum_{l=0}\left(-d_{t}\right)^{l} \frac{\partial L}{\partial q^{a[l]}}=0
$$

Any LF of the form $O(\delta S / \delta q)$ is called an extremal. For any $\operatorname{LF} F\left(q^{[]}\right)$the operation

$$
\frac{d_{\mathrm{EL}} F}{d q^{a}}=\sum_{l=0}^{N_{a}}\left(-\frac{d}{d t}\right)^{l} \frac{\partial F}{\partial q^{a[l]}}
$$

is called the Euler-Lagrange derivative with respect to the coordinate $q^{a}$. One can see that the functional derivative of the action $S$ coincides with the Euler-Lagrange derivative of the Lagrange function,

$$
\frac{\delta S}{\delta q^{a}}=\frac{d_{\mathrm{EL}} L}{d q^{a}}
$$

The Euler-Lagrange derivative has the following property:

$$
\frac{d_{\mathrm{EL}}}{d q^{a}} \frac{d}{d t}=0
$$

To prove this, one may use the relation

$$
\begin{gathered}
\frac{\partial}{\partial q^{a[k]}} \frac{d}{d t}=\frac{\partial}{\partial q^{a[k]}}\left(\partial_{t}+\sum_{l=0} q^{b[l+1]} \frac{\partial}{\partial q^{b[l]}}\right)=\left(1-\delta_{k 0}\right) \frac{\partial}{\partial q^{a[k-1]}} \\
+\left(\partial_{t}+\sum_{l=0} q^{b[l+1]} \frac{\partial}{\partial q^{b[l]}}\right) \frac{\partial}{\partial q^{a[k]}}=\frac{d}{d t} \frac{\partial}{\partial q^{a[k]}}+\left(1-\delta_{k 0}\right) \frac{\partial}{\partial q^{a[k-1]}} .
\end{gathered}
$$

Thus, one gets 


$$
\begin{gathered}
\frac{d_{\mathrm{EL}}}{d q^{a}} \frac{d}{d t}=\sum_{k=0}\left(-\frac{d}{d t}\right)^{k} \frac{\partial}{\partial q^{a[k]}} \frac{d}{d t}=-\sum_{k=0}\left(-\frac{d}{d t}\right)^{k+1} \frac{\partial}{\partial q^{a[k]}}+\sum_{k=1}\left(-\frac{d}{d t}\right)^{k} \frac{\partial}{\partial q^{a[k-1]}} \\
=\frac{d}{d t} \sum_{k=0}\left(-\frac{d}{d t}\right)^{k} \frac{\partial}{\partial q^{a[k]}}-\frac{d}{d t} \sum_{k=1}\left(-\frac{d}{d t}\right)^{k-1} \frac{\partial}{\partial q^{a[k-1]}}=\frac{d}{d t} \frac{d_{\mathrm{EL}}}{d q^{a}}-\frac{d}{d t} \frac{d_{\mathrm{EL}}}{d q^{a}}=0 .
\end{gathered}
$$

\section{B. Noether symmetries}

Consider an infinitesimal inner[8] trajectory variation $\delta q^{a}$ (inner variations vanish together with all their time derivatives at $t_{1}$ and $\left.t_{2}\right)$. Namely,

$$
q^{a}(t) \rightarrow q^{\prime a}(t)=q^{a}(t)+\delta q^{a} .
$$

We suppose that $\delta q^{a}=\delta q^{a}\left(q^{[]}\right)$is an LF. The corresponding first variation of the action can be written as follows:

$$
\delta S={ }_{t_{1}}^{t_{2}} \hat{\delta} L d t
$$

where the operator $\hat{\delta}$, which will be called the transformation operator, acts on the corresponding LF as[9]

$$
\hat{\delta}=\sum_{k=0} \delta q^{a[k]} \frac{\partial}{\partial q^{a[k]}}=\hat{\delta}_{\delta q}
$$

Two simple but useful relations follow from (14):

$$
\hat{\delta} q^{a}=\delta q^{a}, \hat{\delta}_{c^{i} \delta_{i} q}=c^{i} \hat{\delta}_{\delta_{i} q}
$$

The variation (12) is a symmetry transformation of the action $S$, or simply a symmetry of the action $S$, whenever the corresponding first variation of the Lagrange function is reduced to the total time derivative of a LF. Namely, $\delta q$ is a symmetry if

$$
\hat{\delta} L=\frac{d F}{d t}
$$

where $F$ is an LF. In this case the first variation (13) of the action depends on the complete set of the variables $q^{[]}$at $t=t_{1}$ and $t=t_{2}$ only,

$$
\delta S={ }_{t_{1}}^{\mathrm{Z}_{t_{2}}} \hat{\delta} L d t=\left.F\right|_{t_{1}} ^{t_{2}}
$$

Any linear combination of symmetry transformations is a symmetry.

Indeed, let $\delta_{i} q$ be some symmetry transformations, and $\delta q=c^{i} \delta_{i} q$, where $c^{i}$ are some constants. Then, taking into account (15), we obtain:

$$
\hat{\delta}_{\delta_{i} q} L=\frac{d F_{i}}{d t} \Longrightarrow \hat{\delta}_{\delta q} L=\frac{d F}{d t}, F=c^{i} F_{i}
$$

Transformation operators that correspond to symmetry transformations are called symmetry operators.

The above-described symmetry transformations are called Noether symmetries.

Below, we list some properties of the transformation operators and of the symmetry transformations:

a) Any first variation of the Lagrange function can be presented as

$$
\hat{\delta} L=\delta q^{a} \frac{d_{\mathrm{EL}} L}{d q^{a}}+\frac{d P}{d t}=\delta q^{a} \frac{\delta S}{\delta q^{a}}+\frac{d P}{d t},
$$

where $P$ is an LF of the form

$$
P=\sum_{a}^{\prime} \sum_{m=1}^{N_{a}} p_{a}^{m} \delta q^{a[m-1]}, p_{a}^{m}=\sum_{s=l}^{N_{a}}\left(-\frac{d}{d t}\right)^{s-m} \frac{\partial L}{\partial q^{a[s]}} .
$$

One ought to remark that the sum (19) that presents $P$ is running only over those $a$ for which $N_{a}>0$. However, it can be extended over all $a^{\prime}$ s since the momenta $p_{a}^{m}$ that correspond to the degenerate coordinates are zero. Thus, the prime over the sum above can be omitted.

b) Any transformation operator commutes with the total time derivative:

$$
\left[\hat{\delta}, \frac{d}{d t}\right]=0 .
$$

The latter property is justified by the following relations:

$$
\begin{gathered}
\frac{d}{d t} \hat{\delta}=\sum_{k=0}\left[\delta q^{a[k+1]} \frac{\partial}{\partial q^{a[k]}}+\delta q^{a[k]} \frac{\partial}{\partial q^{a[k]}} \partial_{t}\right]+\sum_{k, l=0} q^{b[l+1]} \delta q^{a[k]} \frac{\partial^{2}}{\partial q^{a[k]} \partial q^{b[l]}} \\
\hat{\delta} \frac{d}{d t}=\sum_{l=0}\left[\hat{\delta} q^{b[l+1]}\right] \frac{\partial}{\partial q^{b[l]}}+\hat{\delta} \partial_{t}+\sum_{k, l=0} \delta q^{a[k]} q^{b[l+1]} \frac{\partial^{2}}{\partial q^{b[l]} \partial q^{a[k]}}=\frac{d}{d t} \hat{\delta}
\end{gathered}
$$


c) The commutator of any two transformation operators is a transformation operator as well.

Namely, let $\hat{\delta}_{1} q=\delta q_{1}$, and $\hat{\delta}_{2} q=\delta q_{2}$. Then

$$
\left[\hat{\delta}_{1}, \hat{\delta}_{2}\right]=\hat{\delta}_{3}, \hat{\delta}_{3} q=\hat{\delta}_{1} \delta q_{2}-\hat{\delta}_{2} \delta q_{1}
$$

Indeed, one can write:

$$
\begin{aligned}
& \hat{\delta}_{1} \hat{\delta}_{2}=\sum_{l=0}\left(\hat{\delta}_{1} \delta q_{2}^{b[l]}\right) \frac{\partial}{\partial q^{b[l]}}+\sum_{k, l=0} \delta q_{1}^{a[k]} \delta q_{2}^{b[l]} \frac{\partial}{\partial q^{b[l]}} \frac{\partial}{\partial q^{a[k]}} \\
& =\sum_{l=0} \frac{d^{l}\left(\hat{\delta}_{\varepsilon_{1}} \delta q_{2}^{b}\right)}{d t^{l}} \frac{\partial}{\partial q^{b[l]}}+\sum_{k, l=0} \delta q_{1}^{a[k]} \delta q_{2}^{b[l]} \frac{\partial}{\partial q^{b[l]}} \frac{\partial}{\partial q^{a[k]}}, \quad(22) \\
& \hat{\delta}_{2} \hat{\delta}_{1}=\sum_{k=0}\left(\hat{\delta}_{\varepsilon_{2}} \delta q_{1}^{a[k]}\right) \frac{\partial}{\partial q^{a[k]}}+\sum_{l, k=0} \delta q_{2}^{b[l]} \delta q_{1}^{a[k]} \frac{\partial}{\partial q^{a[k]}} \frac{\partial}{\partial q^{b[l]}} \\
& =\sum_{k=0} \frac{d^{k}\left(\hat{\delta}_{\varepsilon_{2}} \delta q_{1}^{b}\right)}{d t^{k}} \frac{\partial}{\partial q^{a[k]}}+\sum_{k, l=0} \delta q_{1}^{a[k]} \delta q_{2}^{b[l]} \frac{\partial}{\partial q^{b[l]}} \frac{\partial}{\partial q^{a[k]}}
\end{aligned}
$$

Then subtracting Eq. (23) from Eq. (22), we obtain the relation (21).

In other words, the set of all transformation operators form a Lie algebra.

d) The commutator of the Euler-Lagrange derivative and a transformation operator is proportional to the Euler-Lagrange derivative. Namely, if $\hat{\delta} q=\delta q^{b}$, then

$$
\left[\frac{d_{\mathrm{EL}}}{d q^{a}}, \hat{\delta}\right]=\hat{Q}_{a}^{b} \frac{d_{\mathrm{EL}}}{d q^{b}}, \hat{Q}_{a}^{b}=\sum_{k=0}\left(-\frac{d}{d t}\right)^{k} \frac{\partial}{\partial q^{a[k]}} \delta q^{b} .
$$

To prove this property, one may consider a sequence of equalities,

$$
\begin{aligned}
& \mathrm{Z}_{t_{1}} \frac{d_{\mathrm{EL}}(\hat{\delta} F)}{d q^{a}} \zeta^{a} d t={ }_{t_{1}}^{\mathrm{Z}_{2}} \hat{\delta}_{\zeta} \hat{\delta} F d t \\
& ={ }_{t_{1}}^{\mathrm{Z}_{2}} \hat{\delta} \hat{\delta}_{\zeta} F d t+{ }_{t_{1}}^{\mathrm{Z}_{2}} \hat{\delta}_{\hat{\delta}_{\zeta} \delta q} F d t \\
& ={ }_{t_{1}}^{\mathrm{Z}_{2}} \zeta^{a} \sum_{k=0}\left(-\frac{d}{d t}\right)^{k} \hat{\delta} \frac{\partial F}{\partial q^{a[k]}} d t+{ }_{t_{1}}^{\mathrm{Z}_{2}} \hat{\delta}_{\zeta} \delta q^{b} \frac{d_{\mathrm{EL}} F}{d q^{b}} d t \\
& ={ }_{t_{1}}^{\mathrm{Z}_{t_{2}}} \zeta^{a}\left(\hat{\delta} \delta_{a}^{b}+\hat{Q}_{a}^{b}\right) \frac{d_{\mathrm{EL}} F}{d q^{b}} d t, \quad\left(\hat{\delta}_{\zeta} q^{a}=\zeta^{a}\right),
\end{aligned}
$$

where $\zeta(t)$ is an arbitrary inner variation, and $F$ is an LF.

It is useful to keep in mind the following generalization of relation (24):

$$
\left[\left(\frac{d}{d t}\right)^{k} \frac{d_{\mathrm{EL}}}{d q^{a}}, \hat{\delta}\right]=\left(\frac{d}{d t}\right)^{k} \hat{Q}_{a}^{b} \frac{d_{\mathrm{EL}}}{d q^{b}},
$$

which follows immediately from (20) and (24). e) The commutator of two symmetry operators is a symmetry operator as well.

Indeed, let $\hat{\delta}_{1} q=\delta q_{1}$, and $\hat{\delta}_{2} q=\delta q_{2}$ be symmetry transformations, i.e., $\hat{\delta}_{1} L=d F_{1} / d t$, and $\hat{\delta}_{2} L=d F_{2} / d t$. Then, taking into account (20) and (21), we obtain

$$
\left[\hat{\delta}_{1}, \hat{\delta}_{2}\right] L=\hat{\delta}_{3} L=\frac{d}{d t} F_{3}, F_{3}=\hat{\delta}_{1} F_{2}-\hat{\delta}_{2} F_{1}
$$

Thus, the set of symmetry operators of the action $S$ forms a Lie subalgebra of the Lie algebra of all transformation operators.

f) Symmetry transformations transform extremals into extremals.

The validity of this assertion follows from the relations proven below.

Suppose $\hat{\delta}$ is a symmetry operator; then the following relation takes place:

$$
\hat{\delta} \frac{\delta S}{\delta q^{a}}=-\hat{Q}_{a}^{b} \frac{\delta S}{\delta q^{b}} .
$$

Indeed, by virtue of (10), (11), and (24), we can write

$$
\begin{aligned}
& \hat{\delta} \frac{\delta S}{\delta q^{a}}=\hat{\delta} \frac{d_{\mathrm{EL}} L}{d q^{a}}=\frac{d_{\mathrm{EL}}(\hat{\delta} L)}{d q^{a}}-\hat{Q}_{a}^{b} \frac{d_{\mathrm{EL}} L}{d q^{b}} \\
& \quad=\frac{d_{\mathrm{EL}}}{d q^{a}} \frac{d F}{d t}-\hat{Q}_{a}^{b} \frac{\delta S}{\delta q^{b}}=-\hat{Q}_{a}^{b} \frac{\delta S}{\delta q^{b}} .
\end{aligned}
$$

A generalization of (27) based on the relation (24) reads:

$$
\hat{\delta} \frac{d^{k}}{d t^{k}} \frac{\delta S}{\delta q^{a}}=-\frac{d^{k}}{d t^{k}} \hat{Q}_{a}^{b} \frac{\delta S}{\delta q^{b}} .
$$

g) Symmetry transformations transform genuine trajectories into genuine trajectories.

Indeed, suppose that $\tilde{q}^{a}$ be a genuine trajectory, that is

$$
\left.\frac{\delta S}{\delta q^{a}}\right|_{\tilde{q}}=0
$$

and $\delta q^{a}$ be a symmetry transformation. Then the transformed trajectory $\tilde{q}^{\prime a}=\tilde{q}^{a}+\delta q^{a}$ is also a genuine one. Indeed, by virtue of (27) and (29), we get:

$$
\left.\frac{\delta S}{\delta q^{a}}\right|_{\tilde{q}^{\prime}=\tilde{q}+\delta q}=\left.\frac{\delta S}{\delta q^{a}}\right|_{\tilde{q}}+\left.\hat{\delta} \frac{\delta S}{\delta q^{a}}\right|_{\tilde{q}}=\left.\left(\delta_{a}^{b}-\hat{Q}_{a}^{b}\right) \frac{\delta S}{\delta q^{b}}\right|_{\tilde{q}}=0 .
$$

\section{Trivial symmetries}

Below, we are going to describe so-called trivial symmetries transformations, which exist for any action.

A symmetry transformation is called a trivial symmetry transformation whenever the corresponding trajectory variation has the form

$$
\delta q^{a}=\hat{U}^{a b} \frac{\delta S}{\delta q^{b}},
$$


where $\hat{U}$ is an antisymmetric LO, that is $\left(\hat{U}^{T}\right)^{a b}=-\hat{U}^{a b}$. Thus, trivial symmetry transformations do not affect genuine trajectories. (One can prove, see below, that any symmetry transformation that vanishes on the equations of motion, $\delta q^{a}=O(\delta S / \delta q)$, is trivial, namely it has the form (30)). With the help of relations (5) and (18), we can easily verify that (30) is actually a symmetry transformation. Indeed,

$$
\hat{\delta} L=\frac{d_{\mathrm{EL}} L}{d q^{b}} \hat{U}^{a b} \frac{d_{\mathrm{EL}} L}{d q^{b}}+\frac{d P}{d t}=\frac{d F}{d t}+\frac{d P}{d t}=\frac{d(F+P)}{d t},
$$

where $F$ and $P$ are some LF.

Since trivial symmetry transformations are proportional to the equations of motion, they do not change genuine trajectories, as was already mentioned above.

The commutator of a symmetry operator and a trivialsymmetry operator is a trivial-symmetry operator. Namely, if

$$
\hat{\delta}_{1} L=d F_{1} / d t, \hat{\delta}_{2} L=d F_{2} / d t, \hat{\delta}_{2} q^{a}=\delta_{2} q^{a}=\hat{V}^{a b} \delta S / \delta q^{b},
$$

then

$$
\left[\hat{\delta}_{1}, \hat{\delta}_{2}\right] L=\hat{\delta}_{3} L, \hat{\delta}_{3} q^{a}=\delta_{3} q^{a}=\hat{U}^{a b} \frac{\delta S}{\delta q^{b}}
$$

where $\hat{V}^{a b}$ and $\hat{U}^{a b}$ are some antisymmetric LO.

To verify (31), we remark that, according to (21), $\hat{\delta}_{3}$ is a symmetry operator, with $\delta_{3} q=\hat{\delta}_{1} \delta_{2} q-\hat{\delta}_{2} \delta_{1} q$, where $\delta_{1} q=$ $\hat{\delta}_{1} q^{a}$. The term $\hat{\delta}_{1} \delta_{2} q$ can be calculated with the help of (14),

$$
\hat{\delta}_{1} \delta_{2} q^{a}=\sum_{k=0} \frac{\partial\left(\delta_{2} q^{a}\right)}{\partial q^{c[k]}}\left[\frac{d^{k}}{d t^{k}}\left(\hat{V}^{c b} \frac{\delta S}{\delta q^{b}}\right)\right]
$$

and the term $\hat{\delta}_{2} \delta_{1} q$ can be calculated with the help of (27),

$$
\hat{\delta}_{2} \delta_{1} q^{a}=\left(\hat{\delta}_{2} \hat{V}^{a b}\right) \frac{\delta S}{\delta q^{b}}+\hat{V}^{a b} \hat{\delta}_{2} \frac{\delta S}{\delta q^{b}}=\left(\hat{\delta}_{2} \hat{V}^{a b}\right) \frac{\delta S}{\delta q^{b}}-\hat{V}^{a b} \hat{Q}_{b}^{c} \frac{\delta S}{\delta q^{c}}
$$

Thus, we obtain: $\hat{\delta}_{3} q^{a}=\delta_{3} q^{a}=\hat{U}^{a b} \delta S / \delta q^{b}$, where $\hat{U}^{a b}$ is an antisymmetric LO of the form

$$
\hat{U}^{a b}=\sum_{k=0}\left[\frac{\partial\left(\delta_{2} q^{a}\right)}{\partial q^{c[k]}}\left(\frac{d}{d t}\right)^{k} \hat{V}^{c b}+\hat{V}^{a c}\left(-\frac{d}{d t}\right)^{k} \frac{\partial\left(\delta_{2} q^{b}\right)}{\partial q^{c[k]}}\right]-\hat{\delta}_{2} \hat{V}^{a b}
$$

We call two symmetry transformations $\delta_{1} q$ and $\delta_{2} q$ equivalent $\left(\delta_{1} q \sim \delta_{2} q\right)$ whenever they differ by a trivial symmetry transformation:

$$
\delta_{1} q \sim \delta_{2} q \Longleftrightarrow \delta_{1} q^{a}-\delta_{2} q^{a}=\hat{U}^{a b} \frac{\delta S}{\delta q^{b}}
$$

Here $\left(\hat{U}^{T}\right)^{a b}=-\hat{U}^{a b}$.

Let $\mathbf{G}(S)$ be the Lie algebra of all symmetries of the action $S$. The trivial symmetries form the ideal $\mathbf{G}_{\mathrm{tr}}(S)$ in the Lie algebra $\mathbf{G}(S)$. Then the classes of equivalent symmetries form a Lie algebra $\mathbf{G}_{\mathrm{Ph}}(S)$ isomorphic to the quotient algebra:

$$
\mathbf{G}_{\mathrm{Ph}}(S)=\mathbf{G}(S) / \mathbf{G}_{\mathrm{tr}}(S) .
$$

\section{DYNAMICALLY EQUIVALENT ACTIONS}

Very often we encounter an action

$$
S_{\mathrm{E}}[q, y]={ }^{\mathrm{Z}} L_{\mathrm{E}}\left(q^{[]}, y^{[]}\right) d t
$$

which contains two groups of coordinates $q^{[]}$and $y^{[]}$such that the Euler-Lagrange allow one to express all $y$ via $q^{[]}$. It is convenient to call $S_{\mathrm{E}}[q, y]$ the extended action. One can try to eliminate the variables $y$ from the extended action to get some reduced action, which depends now only on $q$, and ask the question: What is the relation between the extended and the reduced actions? There exist a case when this question has a definite answer [2, 5]. Namely, let us suppose that the EulerLagrange $\delta S_{\mathrm{E}}[q, y] / \delta y=0$ allow one to express uniquely the variables $y$ as LF of the variables $q$,

$$
\frac{\delta S_{\mathrm{E}}[q, y]}{\delta y}=0 \Longleftrightarrow y=\bar{y}\left(q^{[]}\right)
$$

Then we define the reduced action $S[q]$

$$
S[q]=S_{\mathrm{E}}[q, \bar{y}]=^{\mathrm{Z}} L_{\mathrm{E}}\left(q^{\square}, \bar{y}^{\square}\right) d t=^{\mathrm{Z}} L\left(q^{[]}\right) d t .
$$

Let us compare the Euler-Lagrange that correspond to both actions. First consider the variation of the reduced action $\delta S$ under arbitrary inner variations $\delta q$, 


$$
\delta S[q]=^{\mathrm{Z}}\left(\left.\frac{\delta S_{\mathrm{E}}[q, y]}{\delta q^{i}}\right|_{y=\bar{y}} \delta q^{i}+\left.\frac{\delta S_{\mathrm{E}}[q, y]}{\delta y^{\alpha}}\right|_{y=\bar{y}} \delta \bar{y}^{\alpha}\right) d t=\frac{\mathrm{Z}}{\delta S[q]} \frac{\delta q^{i}}{\delta q^{i} d t}
$$

In virtue of (34), the Euler-Lagrange of the reduced action read

$$
\frac{\delta S[q]}{\delta q}=\left.\frac{\delta S_{\mathrm{E}}[q, y]}{\delta q}\right|_{y=\bar{y}}=0 .
$$

On the other hand, the Euler-Lagrange of the extended action $S_{\mathrm{E}}[q, y]$ are

$$
\frac{\delta S_{\mathrm{E}}[q, y]}{\delta q}=0, \frac{\delta S_{\mathrm{E}}[q, y]}{\delta y}=0 \Longleftrightarrow y=\bar{y}\left(q^{\square}\right) .
$$

They are reduced to (37) in the $q$-sector. We can see that the extended action and the reduced action lead to the same Euler-Lagrange for $q$. This is why the variables $y$ are called the auxiliary variables. The auxiliary variables $y$ can be eliminated from the action with the help of the Euler-Lagrange. Further, we call the actions $S_{\mathrm{E}}[q, y]$ and $S[q]$ the dynamically equivalent actions.

One ought to stress that the above equivalence is a consequence of the assumption that the variables $y$ are expressed via $q$ by means of the equations $\delta S / \delta y=0$ only. If, for this purpose, some of the equations $\delta S / \delta q=0$ are used as well, then the above equivalence can be absent. Of course, the solutions of the Euler-Lagrange for the reduced action, together with the definition $y=\bar{y}$, contain all solutions of the EulerLagrange for the extended action (as it is easily seen from Eq. (36)). However, the reduced action can imply additional solutions.

Actions containing auxiliary variables and the corresponding reduced actions have similar properties, in particular, there exists a direct relation between their symmetry transformations.

As was mentioned above, we are going to relate the symmetry properties of the extended and reduced actions. To this end, it is convenient to make an invertible coordinate replacement, $\left(q^{a}, y^{\alpha}\right) \rightarrow \tilde{q}^{A}=\left(q^{a}, z^{\alpha}\right), y=z+\bar{y}\left(q^{[l]}\right)$, in the extended action. In fact, we are going to consider a modified extended action $\tilde{S}[\tilde{q}]$, which is obtained from the extended action $S_{\mathrm{E}}[q, y]$ as follows:

$$
\tilde{S}[\tilde{q}]={ }^{\mathrm{Z}} \tilde{L}\left(\tilde{q}^{[]}\right) d t=S_{\mathrm{E}}[q, z+\bar{y}]={ }^{\mathrm{Z}} L_{\mathrm{E}}\left(q^{[]}, z^{[]}+\bar{y}^{[]}\right) d t .
$$

The extended action $S_{\mathrm{E}}[q, y]$ and the modified extended action $\tilde{S}[\tilde{q}]$ are completely equivalent. They lead to completely equivalent Euler-Lagrange. Thus, it is sufficient to study the relation between the symmetry properties of the modified extended action $\tilde{S}[\tilde{q}]$ and the reduced action $S[q]$.

Note that

$$
S[q]=\left.\tilde{S}[\tilde{q}]\right|_{z=0}, L\left(q^{[]}\right)=\left.\tilde{L}\left(\tilde{q}^{[]}\right)\right|_{z=0}
$$

Besides, the action (38) can be presented in the form

$$
\begin{gathered}
\tilde{S}[\tilde{q}]=S[q]+\Delta S[\tilde{q}], \Delta S[\tilde{q}]=\Delta L d t, \\
\quad=L_{\mathrm{E}}\left(q^{[]}, z^{[]}+\bar{y}^{[]}\right)-L_{\mathrm{E}}\left(q^{[]}, \bar{y}^{[]}\right) .
\end{gathered}
$$

The variables $z$ are auxiliary ones for the action $\tilde{S}[\tilde{q}]$, and, in particular, $z=0$ on the Euler-Lagrange. Indeed,

$$
\frac{\delta \tilde{S}[\tilde{q}]}{\delta z}=0 \Longleftrightarrow \frac{\delta S_{\mathrm{E}}[q, y]}{\delta y}=0 \Longrightarrow y=\bar{y}\left(q^{[]}\right) \Longrightarrow z=0 .
$$

The latter implies:

$$
\frac{\delta \tilde{S}}{\delta z^{\alpha}}=\frac{\delta \Delta S}{\delta z^{\alpha}}=\hat{U}_{\alpha \beta} z^{\beta}=0 .
$$

Since equation (41) has the unique solution $z=0$, one can easily verify that $\hat{U}$ is an invertible LO. The equation (42) implies

$$
\Delta L=z^{\alpha} \hat{K}_{\alpha \beta} z^{\beta}+\frac{d}{d t} F,
$$

where $\hat{K}$ is a symmetric LO, and $F$ is an LF. Besides, one can write

$$
z^{\alpha}=\left(\hat{U}^{-1}\right)^{\alpha \beta} \frac{\delta \Delta S}{\delta z^{\beta}}=\left(\hat{U}^{-1}\right)^{\alpha \beta} \frac{\delta \tilde{S}}{\delta z^{\beta}} .
$$

On the other hand, due to the property (11), one can write

$$
\frac{\delta \Delta S}{\delta q^{a}}=\frac{d_{\mathrm{EL}} \Delta L}{d q^{a}}=\frac{d_{\mathrm{EL}}}{d q^{a}}\left[z^{\alpha} \hat{K}_{\alpha \beta} z^{\beta}\right] .
$$

Then, taking into account $(43,44)$, and the definition of the Euler-Lagrange derivative, we get the following useful relation:

$$
\frac{\delta \Delta S}{\delta q^{a}}=\hat{\Lambda}_{a}^{\alpha} \frac{\delta \Delta S}{\delta z^{\alpha}}, \hat{\Lambda}_{a}^{\alpha}=\sum_{l=0}\left(-\frac{d}{d t}\right)^{l} z^{v} \frac{\partial \hat{K}_{v \beta}}{\partial q^{a[l]}}\left(\hat{U}^{-1}\right)^{\beta \alpha},
$$

where $\hat{\Lambda}_{a}^{\alpha}$ is an LO.

\section{SYMMETRIES OF THE EXTENDED AND THE REDUCED ACTIONS}

There exists a one-to-one correspondence (isomorphism) between the symmetry classes of the extended action $\tilde{S}[\tilde{q}]$ and the reduced action $S[q]$. Below, we prove a set of assertions, which justify, in fact, this correspondence. 
i) If the transformation

$$
\delta \tilde{q}^{A}=\left(\begin{array}{c}
\delta^{\prime} q^{a} \\
\delta z^{\alpha}
\end{array}\right)
$$

is a symmetry of the extended action $\tilde{S}$, then the transformation

$$
\delta q^{a}=\left.\delta^{\prime} q\right|_{z=0}
$$

is a symmetry of the reduced action $S$.

Indeed, let (46) be a symmetry of the action $\tilde{S}$. Then

$$
\hat{\delta}_{\delta \tilde{q}} \tilde{L}=\frac{d}{d t} \tilde{F}
$$

where $\tilde{F}$ is an LF. Considering (48) at $z=\delta z=0$, we get

$$
\hat{\delta}_{\delta q} L=\frac{d}{d t} F, \delta q^{a}=\left.\delta^{\prime} q\right|_{z=0}, F=\left.\tilde{F}\right|_{z=0}
$$

where $L$ is given by (39). Thus, any symmetry of the action $\tilde{S}$ implies a symmetry of the action $S$. The symmetry $\delta q$ obtained in such a way can be called the symmetry reduction of the extended action.

ii) If the transformation $\delta q$ is a symmetry of the reduced action $S$, then the transformation

$$
\delta \tilde{q}^{A}=\left(\begin{array}{c}
\delta q^{a} \\
\delta z^{\alpha}
\end{array}\right), \delta z^{\alpha}=-\left(\hat{\Lambda}^{T}\right)_{a}^{\alpha} \delta q^{a}
$$

where the LO $\hat{\Lambda}$ defined by Eq. (45) is a symmetry of the extended action $\tilde{S}$.

To prove this assertion, let us consider the first variation $\hat{\delta}_{\delta \tilde{q}} \tilde{L}$ of the Lagrange function $\tilde{L}$. Since $\delta q$ is a symmetry of the reduced action $S$, the relation $\hat{\delta}_{\delta q} L=d F / d t$, where $F$ is an LF, holds true. Thus, with the help of the property (15), one may write the variation $\hat{\delta}_{\delta \tilde{q}} \tilde{L}$ in the form

$$
\hat{\delta}_{\delta \tilde{q}} \tilde{L}=\left(\hat{\delta}_{\delta q}+\hat{\delta}_{\delta z}\right) \tilde{L}=\frac{d}{d t} F+\left(\hat{\delta}_{\delta q}+\hat{\delta}_{\delta z}\right) \Delta L
$$

Now, we present the variations $\hat{\delta}_{\delta q} \Delta L$ and $\hat{\delta}_{\delta z} \Delta L$ with the help of relation (18). Besides, taking into account the expression (49) for the variation $\delta z$, we get

$$
\hat{\delta}_{\delta \tilde{q}} \tilde{L}=\frac{d}{d t}\left(F+P_{q}+P_{z}\right)+\delta q^{a} \frac{\delta \Delta S}{\delta q^{a}}-\left[\left(\hat{\Lambda}^{T}\right)_{a}^{\alpha} \delta q^{a}\right] \frac{\delta \Delta S}{\delta z^{\alpha}},
$$

where $P_{q}$ and $P_{z}$ are some LF. Using (45) and (5), we may write

$$
\delta q^{a} \frac{\delta \Delta S}{\delta q^{a}}=\delta q^{a} \hat{\Lambda}_{a}^{\alpha} \frac{\delta \Delta S}{\delta z^{\alpha}}=\left[\left(\hat{\Lambda}^{T}\right)_{a}^{\alpha} \delta q^{a}\right] \frac{\delta \Delta S}{\delta z^{\alpha}}+\frac{d G}{d t}
$$

where $G$ is an LF. Thus, the variation $\hat{\delta}_{\delta \tilde{q}} \tilde{L}$ is reduced to the total derivative of an LF,

$$
\hat{\delta}_{\delta \tilde{q}} \tilde{L}=\frac{d}{d t}\left(F+P_{q}+P_{z}+G\right) .
$$

Thus, $\delta \tilde{q}$ is a symmetry of the extended action $\tilde{S}$.

iii) Any symmetry of the form

$$
\delta \tilde{q}=\left(\begin{array}{c}
0 \\
\delta z
\end{array}\right)
$$

of the extended action $\tilde{S}$ is trivial.

Since $\delta \tilde{q}$ is a symmetry of the action $\tilde{S}$, one can write

$$
\hat{\delta}_{\delta \tilde{q}} \tilde{L}=\hat{\delta}_{\delta z} \tilde{L}=\frac{d F}{d t},
$$

where $F$ is an LF. Taking into account (18), we may rewrite Eq. (54) as

$$
\delta z^{\alpha} \frac{\delta \tilde{S}}{\delta z^{\alpha}}=\frac{d F^{\prime}}{d t},
$$

where $F^{\prime}$ is an LF. The left-hand side of equation (55) can be transformed, with the help of (42) and (5), to the form

$$
\delta z^{\alpha} \frac{\delta \tilde{S}}{\delta z^{\alpha}}=\delta z^{\alpha} \hat{U}_{\alpha \beta} z^{\beta}=\left[\left(\hat{U}^{T}\right)_{\beta \alpha} \delta z^{a}\right] z^{\beta}+\frac{d F^{\prime \prime}}{d t},
$$

where $F^{\prime \prime}$ is an LF. Thus, the equation (55) may be reduced to

$$
z^{\beta} f_{\beta}=\frac{d \Phi}{d t}, f_{\beta}=\left(\hat{U}^{T}\right)_{\beta \alpha} \delta z^{a},
$$

where $f\left(Q^{[]}\right)$and $\Phi\left(Q^{\square}\right)$ are some LF. Let us present the $\mathrm{LF} \Phi$ as

$$
\begin{aligned}
& \Phi\left(Q^{[]}\right)=\Phi_{0}\left(q^{[]}\right)+\Phi_{1}\left(Q^{[]}\right), \\
& \Phi_{0}=\left.\Phi\right|_{z=0},\left.\Phi_{1}\right|_{z=0}=\sum_{k=0}^{N} \Phi_{\alpha(k)}\left(Q^{[]}\right) z^{\alpha[k]}, N<\infty .
\end{aligned}
$$

It follows from equation (56) that $d \Phi_{0} / d t \equiv 0$. According to (6), the latter implies $\Phi_{0} \equiv$ const. From (56), we get the equation

$$
\sum_{k=0}^{N+1} \varphi_{\alpha(k)} z^{\alpha[k]}=0
$$

where

$$
\begin{aligned}
& \varphi_{\alpha(0)}=f_{\alpha}-\dot{\Phi}_{\alpha(0)}, \varphi_{\alpha(N+1)}=-\Phi_{\alpha(N)}, \\
& \varphi_{\alpha(k)}=-\left[\Phi_{\alpha(k-1)}+\dot{\Phi}_{\alpha(k)}\right], k=1, \ldots, N .
\end{aligned}
$$

The general solution of Eq. (58) is

$$
\varphi_{\alpha(k)}=\sum_{l=0}^{N+1} m_{\alpha(k) \mid \beta(s) l} z^{\beta[s]}, m_{\alpha(k) \mid \beta(s) l}=-m_{\beta(s) \mid l \alpha(k)},
$$

where $m_{\alpha(k) \mid \beta(s) l}\left(Q^{[]}\right)$are some LF. Then the LF $\Phi_{\alpha(k)}$ and $f_{\alpha}$ can be found from Eq. (59):

$$
\begin{aligned}
& \Phi_{\alpha(k)}=-\sum_{m=0}^{N-k} \sum_{l=0}^{N+1}\left(-\frac{d}{d t}\right)^{m}\left[m_{\alpha(k+m+1) \mid \beta(l)} z^{\beta[l]}\right], \\
& f_{\alpha}=\sum_{m, l=0}^{N+1}\left(-\frac{d}{d t}\right)^{m}\left[m_{\alpha(m) \mid \beta(l)} z^{\beta[l]}\right] \equiv \hat{m}_{\alpha \beta} z^{\beta},
\end{aligned}
$$


where $\hat{m}_{\alpha \beta}$ is an antisymmetric LO. Thus, we get from (56)

$$
\delta z^{\alpha}=\hat{M}^{\alpha \beta} \frac{\delta \tilde{S}}{\delta z^{\beta}}, \hat{M}^{\alpha \beta}=\left[\left(\hat{U}^{T}\right)^{-1}\right]^{\alpha \gamma} \hat{m}_{\gamma \delta}\left(\hat{U}^{-1}\right)^{\delta \beta}
$$

where $\hat{M}^{\alpha \beta}$ is an antisymmetric LO. Therefore, the symmetry (53) is trivial.

iv) Suppose both transformations $\delta \tilde{q}_{1}$ and $\delta \tilde{q}_{2}$ to be symmetries of the extended action $\tilde{S}$ such that their reductions coincide, that is

$$
\left.\delta^{\prime} q_{1}\right|_{z=0}=\left.\delta^{\prime} q_{2}\right|_{z=0}=\delta q .
$$

Then these symmetries are equivalent,

$$
\delta \tilde{q}_{1} \sim \delta \tilde{q}_{2}
$$

which means that $\delta \tilde{q}_{1}$ and $\delta \tilde{q}_{2}$ differ by a trivial symmetry.

Thus, we have to prove that the transformation

$$
\Delta \tilde{q}=\delta \tilde{q}_{1}-\delta \tilde{q}_{2}=\left(\begin{array}{c}
\Delta q^{\prime}=\delta^{\prime} q_{1}-\delta^{\prime} q_{2} \\
\Delta z=\delta z_{1}-\delta z_{2}
\end{array}\right),\left.\Delta q^{\prime}\right|_{z=0}=0
$$

is a trivial symmetry of the extended action $\tilde{S}$. In virtue of Eq. (63), the LF $\Delta q^{\prime}$ may be presented as

$$
\Delta q^{\prime a}=\hat{m}_{\alpha}^{a} z^{\alpha},
$$

where $\hat{m}$ is an LO. With the help of (44), we get for $\Delta q^{\prime}$ the following expression:

$$
\Delta q^{\prime a}=\hat{M}^{a \beta} \frac{\delta \tilde{S}}{\delta z^{\beta}}
$$

where $\hat{M}=\hat{m} \hat{U}^{-1}$ is an LO.

Let us present the transformation $\Delta \tilde{q}$ in the form $\Delta \tilde{q}=$ $\Delta_{1} \tilde{q}+\Delta_{2} \tilde{q}$, where

$$
\Delta_{1} \tilde{q}=\hat{M}^{A B} \frac{\delta \tilde{S}}{\delta \tilde{q}^{B}}, \hat{M}^{A B}=\left(\begin{array}{cc}
0 & \hat{M}^{a \beta} \\
-\left(\hat{M}^{T}\right)^{\alpha b} & 0
\end{array}\right),
$$

and

$$
\Delta_{2} \tilde{q}=\left(\begin{array}{c}
0 \\
\Delta \sigma^{\prime \prime}
\end{array}\right)
$$

The transformations $\Delta_{1} \tilde{q}$ is a trivial symmetry since the LO $\hat{M}^{A B}$ is antisymmetric, that is $\left(\hat{M}^{T}\right)^{A B}=-\hat{M}^{A B}$. Thus, $\Delta_{2} \tilde{q}$ is a symmetry of the extended action $\tilde{S}$. Besides, the latter symmetry has a special form (68). It was proven in item c) that any symmetry of such a form is trivial. Therefore, the symmetry $\Delta \tilde{q}$ is trivial as well.

v) Let a transformation $\delta \tilde{q}$ be a trivial symmetry of the extended action $\tilde{S}$. Then its reduction $\delta q$ is a trivial symmetry of the reduced action $S$.

According to this assumption, we may write

$$
\delta \tilde{q}^{A}=\left(\begin{array}{l}
\delta^{\prime} q^{a}=\hat{M}^{a b} \frac{\delta \tilde{S}}{\delta q^{b}}+\hat{M}^{a \beta} \frac{\delta \tilde{S}}{\delta z^{\beta}} \\
\delta z^{\alpha}=-\left(\hat{M}^{T}\right)^{b \alpha} \frac{\delta \tilde{S}}{\delta q^{b}}+\hat{M}^{\alpha \beta} \frac{\delta \tilde{S}}{\delta z^{\beta}}
\end{array}\right),
$$

where the local operators $\hat{M}^{a b}$ and $\hat{M}^{\alpha \beta}$ are antisymmetric. Then the reduction $\delta q=\left.\delta^{\prime} q\right|_{z=0}$ of the transformation (69) reads

$$
\delta q^{a}=\hat{m}^{a b} \frac{\delta S}{\delta q^{b}}, \hat{m}^{a b}=\left.\hat{M}^{a b}\right|_{z=0} .
$$

The LO $\hat{m}^{a b}$ is antisymmetric. Thus, (70) is a trivial symmetry of the reduced action $S$.

vi) Let a symmetry $\delta q$ of a reduced action $S$ be trivial. Then any extension of this symmetry to the symmetry $\delta \tilde{q}$ of the extended action $\tilde{S}$ is trivial as well.

Since $\delta q$ is a trivial symmetry, one can write

$$
\delta q^{a}=\hat{m}^{a b} \frac{\delta S}{\delta q^{b}}
$$

where $\hat{m}^{a b}$ is an antisymmetric LO. Consider the following extension of the symmetry $\delta q^{a}$ :

$$
\delta \tilde{q}_{1}=\left(\begin{array}{c}
\delta^{\prime} q \\
0
\end{array}\right), \delta^{\prime} q^{a}=\hat{m}^{a b} \frac{\delta \tilde{S}}{\delta q^{b}},
$$

which is a trivial symmetry of the extended action $\tilde{S}$. Any other extension of $\delta q$ differs from $\delta \tilde{q}_{1}$ by a trivial symmetry, according to item (iv). Therefore, any extension of the trivial symmetry is a trivial symmetry as well.

Concluding, we can see that there exists an isomorphism between classes of equivalent symmetries of dynamically equivalent actions. Since the Lagrangian and Hamiltonian actions are dynamically equivalent, one can study the symmetry structure of any singular theory considering the first-order Hamiltonian action.

\section{Acknowledgement}

Gitman is grateful to the foundations FAPESP, CNPq for permanent support and to the Lebedev Physics Institute (Moscow) for hospitality; Tyutin thanks RFBR 05-02-17217 and LSS-1578-2003.2 for partial support.
[1] P. G. Bergmann, Phys. Rev. 75, 680 (1949); P.M. Dirac, Can. J. Math. 2, 129 (1950); Lectures on Quantum Mechanics (Yeshiva University, New York 1964); E. C. G. Sudarshan, N. Mukunda,
Classical Dynamics: A Modern Perspective (Wiley, New York 1974); D. M. Gitman and I.V. Tyutin, Quantization of Fields with Constraints (Springer-Verlag, Berlin 1990); M. Henneaux 
and C. Teitelboim, Quantization of Gauge Systems (Princeton University Press, Princeton 1992)

[2] V. A. Borochov and I. V. Tyutin, Physics of Atomic Nuclei, 61, 1603 (1998); ibid 62, 1070 (1999).

[3] D. M. Gitman and I. V. Tyutin, Resenhas IME-USP, v.6, U21162/3, 187 (2004); J. Phys. A 38, 5581 (2005).

[4] D. M. Gitman and I. V. Tyutin, Nucl. Phys. B 630, 509 (2002).

[5] M. Henneaux, Phys. Lett. B238, 299 (1990); I. A. Batalin and I. V. Tyutin, Int. J. Mod. Phys. A11, 1352 (1996); D. M. Gitman, and I. V. Tyutin, Nucl. Phys. B 630, 509 (2002).
[6] The functions $F$ may depend on time explicitly, however, we do not include $t$ in the arguments of the functions.

[7] The functions $L$ may depend on time explicitly, however, we do not include $t$ in the arguments of the functions.

[8] Inner variations vanish together with all their time derivatives at $t_{1}$ and $t_{2}$.

[9] Sometimes, we mark the transformation operator below by the corresponding variation. 